\title{
Letter
}

\section{Interaction of an Ultra-Short Laser Pulse and Ultra-Intense with a Dielectric}

\author{
Oussama Boultif, Slimen Belghit, Beddiaf Zaidi, Abdelaziz Sid \\ PRIMALAB Laboratory, Department of Physics, Faculty of Material Sciences, University of Batna1, Batna, Algeria \\ Email address: \\ belghit.slimen@gmail.com (S. Belghit)
}

To cite this article:

Oussama Boultif, Slimen Belghit, Beddiaf Zaidi, Abdelaziz Sid. Interaction of an Ultra-Short Laser Pulse and Ultra-Intense with a Dielectric. American Journal of Nanosciences. Vol. 4, No. 3, 2018, pp. 26-34. doi: 10.11648/j.ajn.20180403.11

Received: September 6, 2018; Accepted: September 17, 2018; Published: November 7, 2018

\begin{abstract}
In this work, we dedicated to the presentation of our results concerning the evolution of the electron density in the conduction band and the optical damage threshold (OBT). A study of the influence of the initial electronic density on the electron density in the conduction band and on the threshold of optical damage will also be presented. The main objective of this work is the theoretical study of optical damage of dielectric Materials like: Silicate by using a technique based on ultra-short and high-density laser pulses. The mains theoretical models about the OBT technique given in the literature are also studied. A new theoretical model with several improvements is proposed. This model takes into account the recombination's mechanism with three holes. New numerical software has been developed in order to solve the PDE systems of our theoretical model using MATLAB simulation. The contribution of different mechanisms with OBT has been studied numerically. The obtained results showed that recombination mechanism with three holes plays an important role to estimate the density of free electrons and the OBT. We showed in our model that the recombination mechanisms reduce the electron density in the band of conduction is therefore increasing the threshold of optical damage (OBT). The predictions of the code developed in this study have been successfully compared to different experimental measurements of thresholds of breakdown in silica. The found results have a good agreement with experimental results.
\end{abstract}

Keywords: Dielectrics, Optical Breakdown Threshold, Ultra-Short Laser Pulse, Laser-Dielectric Interaction

\section{Introduction}

The interaction of ultra-short laser pulse and ultra-intense with a dielectric is a very large area and a complex [1], since several mechanisms can be active at the same time during the interaction, while being coupled to each other. The overall effect of photoionization may be the result of a mixture of different mechanisms [2]. If taking into account the fact that the pulse contains a limited amount of energy, and also the fact that the two processes of ionization (multi-photon and the avalanche) consume energy $[3,4]$, we can conclude that, in if they were active at the same time, they would be competition.

In addition to the ionization mechanisms cannot neglect the effect of the mechanisms of recombination on the electron density in the conduction band, and then in our model all the mechanisms of ionization and recombination presented above are taken into account $[5,6,7]$.

In this work, we will present the computational results concerning: the electron density in the conduction band, the optical damage threshold and the influence of the initial electronic density on the results.

\section{Calculation of the Electronic Density as a Function of Time}

Our goal in this paragraph is to see more detail how the ionization mechanisms and recombination contribute in the context of ultra-short laser beams [1]. For this reason, we have reserved two paragraphs where we will examine them separately and with more attention. 


\subsection{Calculation of the Electron Density Under the Effect of Ionization Mechanisms}

\subsubsection{Calculation of the Electron Density Under the Effect of Multi-Photon Ionization}

In this paragraph we will treat the influence of the MPI (multi-photon ionization) on the evolution of the density electronics, for that we will solve the Stuart equation taking into account only multi-photon ionization $[3,8]$ :

$$
\frac{d n_{e}(t)}{d t}=w_{m p i}=\sigma_{(k)} I(t)^{k}
$$

Where $w_{m p i}$ : is the term relating to multiphoton ionization. we represent the evolution of the electron density as a function of time under the effect of the MPI only, in two cases, In (Figure 1) and (Figure 2).

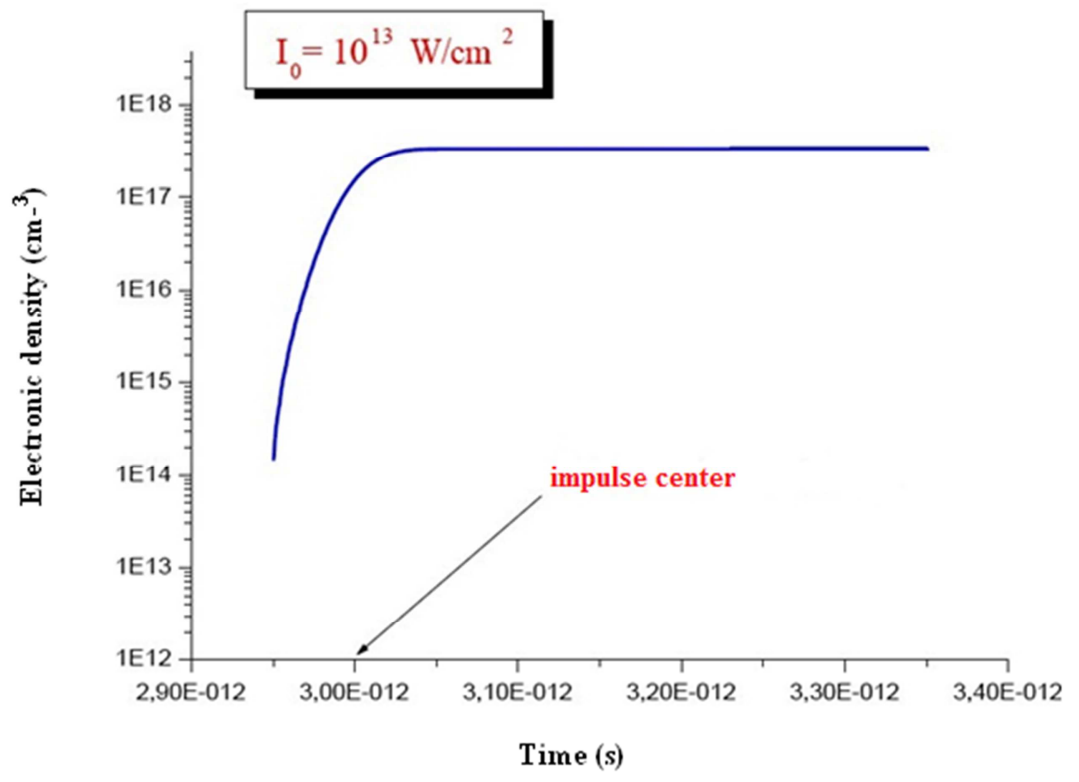

Figure 1. Evolution of the electron density generated by multi-photon ionization as function of the time. For: $\tau_{p}=100 \mathrm{fs} ; \lambda_{p}=800 \mathrm{~nm}, \tau_{p}=100 \mathrm{fs}$ et $I_{0}=$ $10^{13} \mathrm{~W} / \mathrm{cm}^{2}$.

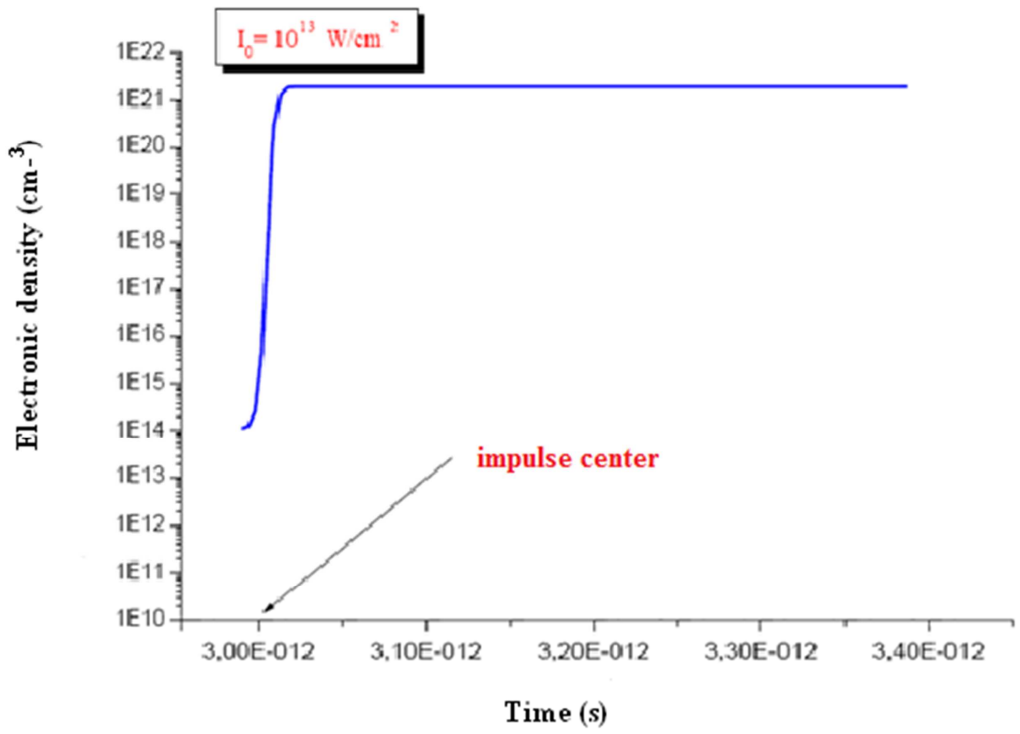

Figure 2. Evolution of the electron density generated by multi-photon ionization as function of the time. For $\tau_{p}=30 \mathrm{fs} . \lambda_{p}=800 \mathrm{~nm}$ et $I_{0}=10^{13} \mathrm{~W} / \mathrm{cm}^{2}$.

The MPI is a process due to the direct action of the laser field on the atoms constituting the medium, the electrons being ejected as a result of this direct interaction, the MPI occurs at timescales of the order of the femtosecond (an almost instantaneous mechanism). So it's a very fast process [1]. From (Figure 1), it is noted that the electron density provided by the MPI is of the order of $\sim 10^{17} \mathrm{~cm}^{-3}$. Then it can be concluded that the MPI only plays the role of a primary electron source for the electronic avalanche. For pulses shorter than $(<30 \mathrm{fs})$. MPI becomes the important mechanism. MPI alone can provide the critical electronic density (Figure 2). On the other hand, according to equation (1), the MPI depends strongly on $\alpha I(t)$, then for high intensities when the intensity increases $I>10^{13} \mathrm{~W} / \mathrm{cm}^{2}$. 


\subsubsection{Calculation of the Electron Density Under the Effect of Avalanche Ionization}

AI (avalanche ionization) is a free electron amplifier process [4]. For this process of AI to be able to start and develop, a minimum of material density is required. This process becomes important in the condensed phase, as is the case in the silica that is the subject of our study. To better understand this mechanism, we will solve the Stuart equation taking into account only avalanche ionization, hence:

$$
\frac{d n_{e}(t)}{d t}=w_{A I}=\alpha I(t) n_{e}(t)
$$

Where $w_{A I}$ : is the term of creation of electrons by avalanche.

(Figure 3) shows the evolution of electronic density versus time under AI only.

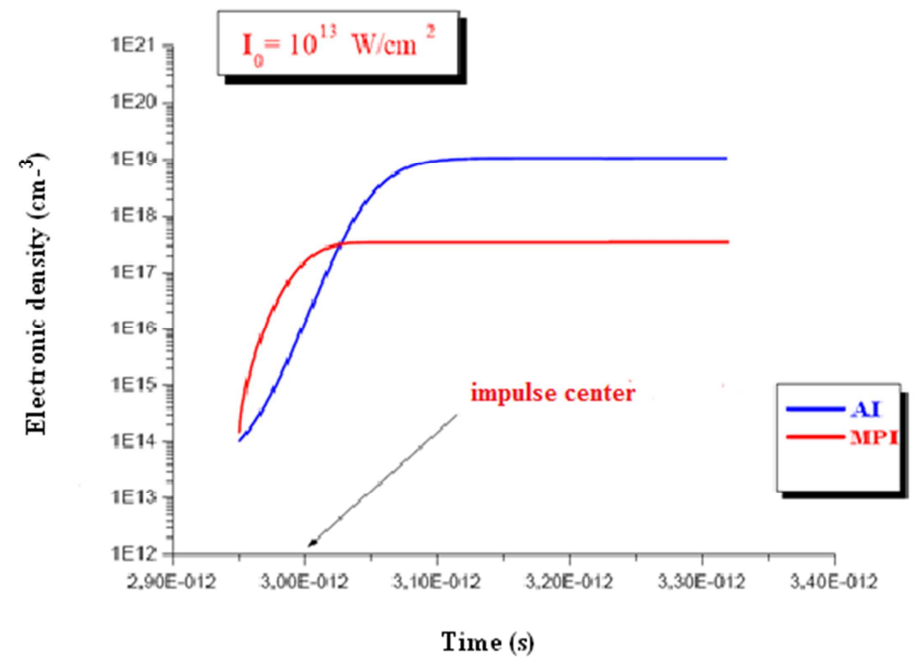

Figure 3. Evolution of the electron density generated by avalanche ionization and multi-photon ionization as function of the time For: $\mathrm{I}_{0}=10^{13} \mathrm{~W} / \mathrm{cm}^{2}, \lambda_{p}=$ $800 \mathrm{~nm}, \tau_{p}=100 \mathrm{fs}$.

The AI comes from collisions between quasi-free electrons, heated by the intense EM field generated by the laser pulse, with the atoms or molecules constituting the material. In the case of an ultra-short pulse propagating in the dielectric medium, the first electrons in the conduction band are produced by the front of the pulse and then heated and excited by the tail of the pulse. Then the impulse has the time to accelerate them to higher energy levels but the multiplication of these electrons by avalanche effect is done after the passage of the impulse (Figure 3 ). So the AI needs some time to develop. On the other hand, the electronic density supplied by the AI is of the order of $10^{19}$ $\mathrm{cm}^{-3}$, for laser intensity of the order of $10^{13} \mathrm{~W}$. It can be concluded that this process does not need much intensity to trigger; however, it needs time to develop.

\subsubsection{Calculation of the Electron Density Under the Effect of Multi-Photon Ionization and Avalanche Ionization}

In this paragraph we will study the case where both mechanisms are active and this amounts to solving the Stuart equation [3]:

$$
\frac{d n_{e}(t)}{d t}=w_{A I}+w_{m p i}=\alpha I(t) n_{e}(t)+\sigma_{(k)} I(t)^{k}
$$

(Figure 4) shows the evolution of the electron density as a function of time under the effect of AI and MPI.

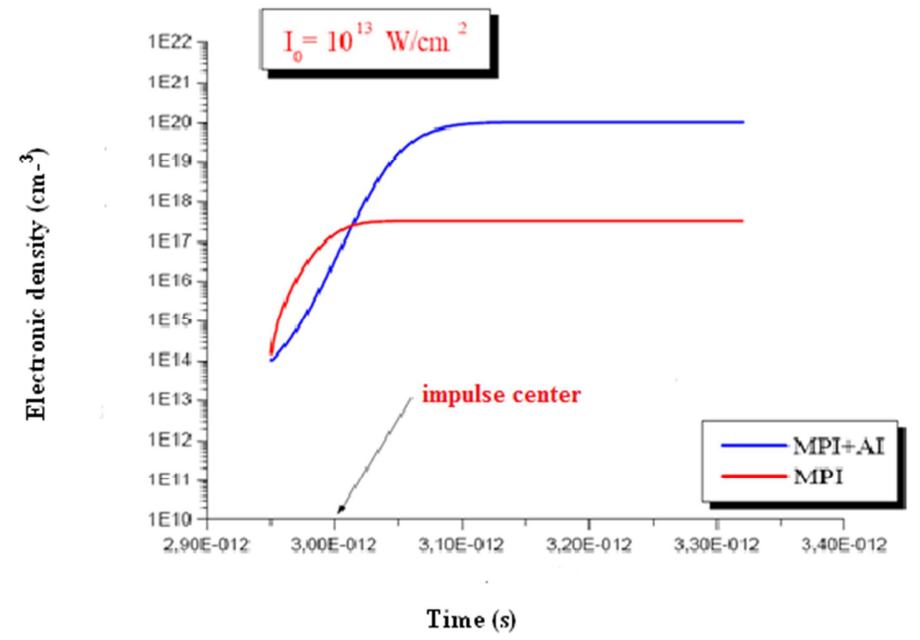

Figure 4. Evolution of the electronic density as function of the time for: $\mathrm{I}_{0}=10^{13} \mathrm{~W} / \mathrm{cm}^{2}, \lambda_{p}=800 \mathrm{~nm}, \tau_{p}=100 \mathrm{fs}$. In addition to the total electron density (blue line), the density generated by multi-photon ionization is also represented (red line). 
For laser pulses $f_{S}$ and for intensities of the order of $10^{13} \mathrm{~W} / \mathrm{cm}^{2}$, our model shows that the MPI will be disadvantaged compared to the IA. It means that at this time scale, the vast majority of free electrons were produced as a result of indirect extraction by the electromagnetic field (AI). But it is important to note that the MPI plays a precursory role important to enable the IA to develop.

\subsection{Calculation of the Electron Density Under the Effect of the Ionization Mechanisms and Recombination}

\subsubsection{Calculation of the Electron Density Under the Effect of the Ionization Mechanisms and Three-Body Recombination}

This section will study the influence of three-body recombination on the evolution of electron density. For this purpose, the Stuart equation supplemented by a three-body recombination term will be solved, to our knowledge; no comparable investigation has yet been made [5].

$$
\frac{d n_{e}(t)}{d t}=w_{A I}+w_{m p i}+\left(\frac{d n_{e}(t)}{d t}\right)_{3 \operatorname{corp}}
$$

With:

$$
\left(\frac{d n_{e}(t)}{d t}\right)_{3 \operatorname{corp}}=-8.75 * 10^{-27} T_{e}^{-\frac{9}{2}} * n_{2}^{3}\left(\frac{c m^{-3}}{s}\right) \text { and } T_{e}=5 \mathrm{eV}
$$

(Figure 5) shows the evolution of electron density as a function of time under the effect of AI and MPI taking into account the three-body recombination.

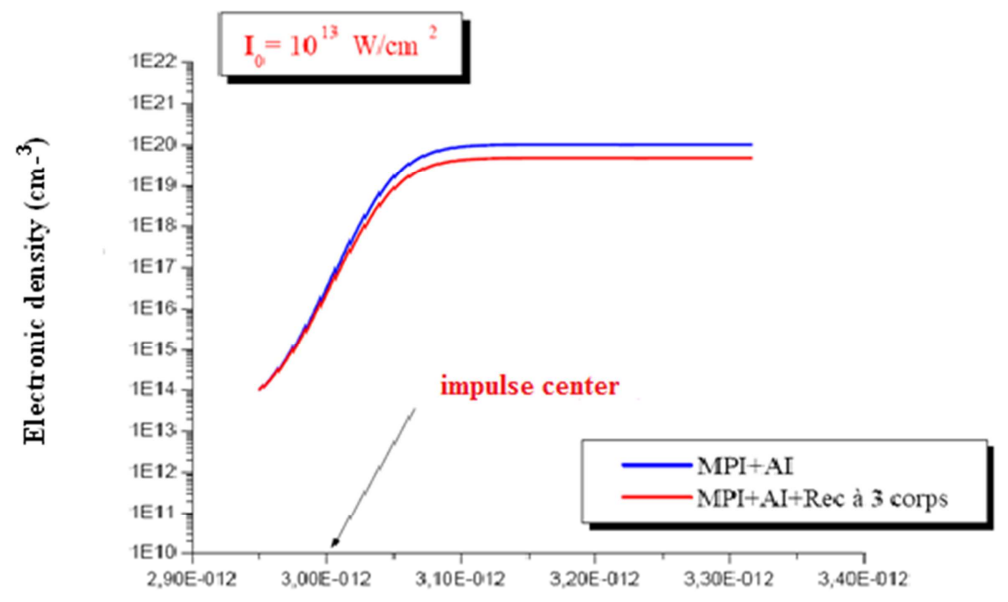

Time (s)

Figure 5. Evolution of the electronic density as function of the time for: $\tau_{\mathrm{p}}=100 \mathrm{fs} ; \mathrm{I}_{0}=10^{13} \mathrm{~W} / \mathrm{cm}^{2}, \lambda_{p}=800$ nm. In addition to the total electron density (blue line), the influence of three-body recombination is also represented (red line).

It can be seen from (Figure 5) that the exponential increase in electron density is abruptly curbed by three-body recombination [5]. The three-body recombination losses can no longer compensate the electron gain but they reduce the total electron density in the rising edge of the laser pulse. It is also noted that the effect of the three-body recombination becomes important in the falling edge of the laser pulse. During this phase the 3-body recombination losses become large and the electron density decreases rapidly at the end of the laser pulse. Then we conclude that the term that introduced in our model has a significant effect on the evolution of electronic density.

\subsubsection{Calculation of the Electron Density Under the Effect of Ionization Mechanisms and of Excitonic Recombination}

The purpose of this section is to study the influence of self-trapped exciting formation on the evolution of electron density. For this, we will couple Stuart's equation with the evolution equation of self-trapped exciting [7]:

$$
\frac{d n_{e}(t)}{d t}=w_{A I}+w_{m p i}-\frac{d n_{\text {exciton }}}{d t}
$$

With

$\frac{d n_{\text {exction }}}{d t}=-\alpha_{x} I(t) n_{\text {exciton }}(t)-\sigma_{x} \frac{n_{\text {exciton }}}{n_{\text {atom }}} I^{m_{x}}+\frac{n_{e}}{\tau_{t r}}$

Where:

$n_{\text {excition }}$ : is the density of self-trapped excitons, $n_{\text {atom }}$ : is the electronic density of silica.

$\alpha_{x}$ : is the avalanche coefficient of the excitons, $\sigma_{x}$ : is the cross section of the multi-photon absorption of excitons, $\tau_{t r}$ : is the trapping rate, $m_{x}$ : is the number of photons absorbed by the excitons to pass to the bung of conduction. 
To our knowledge, the influence of the formation of self-trapped excitons on the evolution of the electron density has not yet been studied. (Figure 6) shows the evolution of the electron density as a function of time under the effect of AI and MPI taking into account the excitonic recombination.

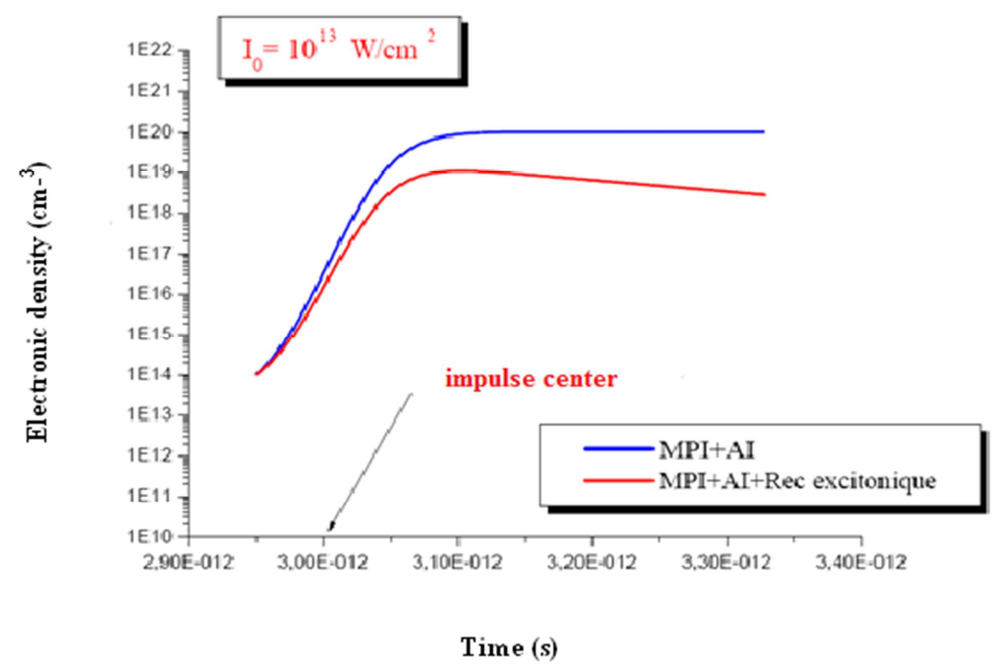

Figure 6. Evolution of the electronic density as function of the time for: $\tau_{\mathrm{p}}=100 \mathrm{fs} ; \mathrm{I}_{0}=10^{13} \mathrm{~W} / \mathrm{cm}^{2}, \lambda_{\mathrm{p}}=800 \mathrm{~nm}$. In addition to the total electron density (blue line), the influence of the excitonic recombination is also represented (red line).

When the electron density near $\left(\sim 10^{20} \mathrm{~cm}^{-3}\right)$, the exponential increase of this density is abruptly curbed by the excitonic recombination. During this phase, a balance between the terms of gain and loss of electrons is established, and the density then follows the temporal evolution of the profile of the laser pulse. In the falling edge of the laser pulse, the losses by excitonic recombination can no longer be compensated for by the gain of electrons, and the density decreases rapidly. It can also be noted that the evolution of the electronic density in the second part of the laser pulse is strongly modified, the electron density decreases rapidly at the end of the laser pulse.

\subsubsection{Calculation of the Electronic Density by Taking into Account all the Mechanisms}

In this section will study the influence of all the mechanisms of ionizations and recombination's on the evolution of the electronic density is for this will solve the system of two equations following $[9,10,11,12,13]$ :

$$
\frac{d n_{e}(t)}{d t}=w_{A I}+w_{m p i}+\left(\frac{d n_{e}(t)}{d t}\right)_{3 \operatorname{corp}}-\frac{d n_{\text {excition }}}{d t}
$$

With

$$
\frac{d n_{\text {excition }}}{d t}=-\alpha_{x} I(t) n_{\text {excition }}(t)-\sigma_{x} \frac{n_{\text {excition }}}{n_{\text {atom }}} I^{m_{x}}+\frac{n_{e}}{\tau_{t r}}
$$

(Figure 7) shows the evolution of the electron density as a function of time under the effect of AI and MPI taking into account the excitonic recombination.

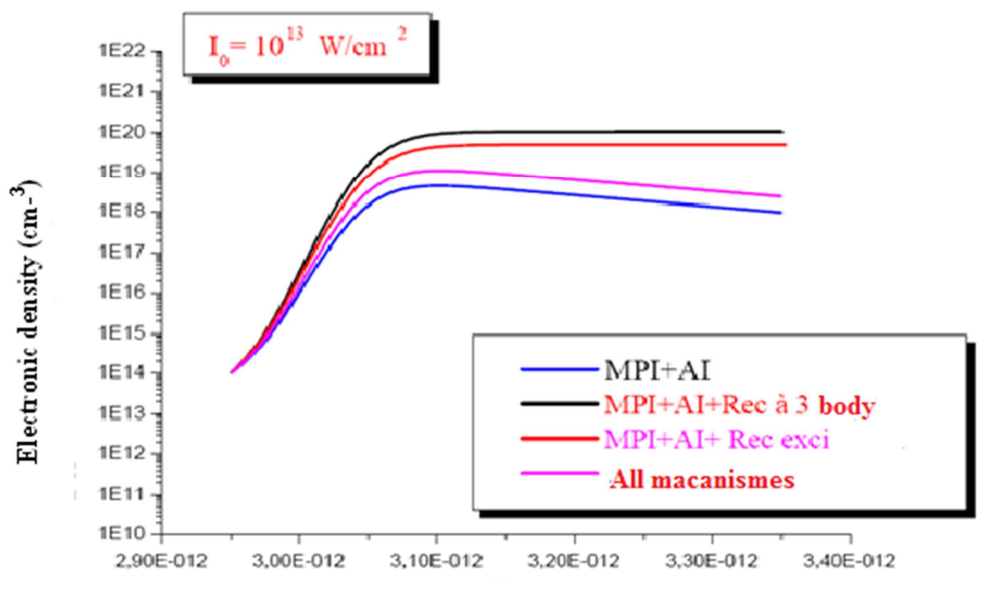

Time (s)

Figure 7. Evolution of the electronic density as function of the time for: $\tau_{\mathrm{p}}=100 \mathrm{fs} ; \mathrm{I}_{0}=10^{13} \mathrm{~W} / \mathrm{cm}^{2}, \lambda_{\mathrm{p}}=800 \mathrm{~nm}$. In four cases: a) MPI+AI (black line); b) $M P I+A I+$ three-body recombination (red line);c) $M P I+A I+$ Exciton (purple line, d) all mechanisms (blue line). 
Note that in the case where both recombination mechanisms are active, the electron density decreases sharply. Note that the excitonic recombination is more important than the 3-body recombination but the latter remains non-negligible.

\section{Calculation of the OBT}

The Optical Breakdown Threshold (OBT) or optical damage threshold is defined as the laser intensity required for the maximum electron density obtained during the duration of the laser pulse to reach the critical electronic breakdown density $[4,14]$. Our goal in this section and to see the effect of the recombination terms that introduced it on the threshold of optical damage. To validate the behavior of our model, we compared its predications with different experimental and numerical values of breakdown thresholds obtained in silica. The relative importance of the different phenomena involved in the breakdown process is compared as a function of the laser pulse duration.

\subsection{Calculation Method}

In order to determine the intensity required to generate breakdown plasma, under certain laser irradiation conditions ( $\tau_{p}$ et $\lambda$ fixed), equation (6) is integrated numerically by the Runge-Kutta method [15] of order 4 . The value of the incident laser intensity is then varied until the maximum electron density obtained during the duration of the laser pulse reaches the critical electronic breakdown density. The intensity for which this equality is verified is then defined as the optical intensity breakdown threshold (OBT). We recall that the condition is defined by:

$$
\omega_{\text {laser }}=\omega_{\text {plasma }}\left(n_{c r}\right) \Rightarrow n_{c r}=\frac{m_{e} \varepsilon_{0} \omega_{\text {laser }}^{2}}{e^{2}}
$$

It is assumed that the initial electron density is $10^{14} \mathrm{~cm}^{-3}$.

\subsection{Calculation of the OBT According to the Duration of the Pulse}

In what follows will present our results of the threshold of optical breakdown in intensity.

\subsubsection{The Results Obtained with a Three-Body Recombination Term}

In Table.1, the thresholds of breakdown determined by our code are compared with the experimental measurements: the first threshold is calculated without taking into account the losses by recombination ( in the model of Stuart et al $n_{e}(0)=$ $10^{14} \mathrm{~cm}^{-3}$ ) [3], and the second is calculated by estimating these losses by three-body recombination using the formula (4).

Table 1. Comparison of the results of the breakdown code with different experimental measurements in silica in two cases: Without recombination 's and with three-body Recombination. All the intensity thresholds are given in $W / \mathrm{cm}^{2}$.

\begin{tabular}{|c|c|c|c|c|c|}
\hline Réf & $\lambda(\mathrm{nm})$ & $\tau_{\mathrm{p}}$ & $\mathbf{I}_{\text {exp }}$ & $\mathbf{I}_{\text {code }}$ (Without Recombination's) & $I_{\text {code }}($ with Rec.3 body) \\
\hline$[16]$ & 800 & $100 \mathrm{fs}$ & $3.83 \times 10^{13}$ & $2.1 \times 10^{13}$ & $2.2 \times 10^{13}$ \\
\hline$[16]$ & 800 & $110 \mathrm{fs}$ & $3.2 \times 10^{13}$ & $1.8 \times 10^{13}$ & $2.1 \times 10^{13}$ \\
\hline$[14]$ & 800 & $140 \mathrm{fs}$ & $1.945 \times 10^{13}$ & $1.69 \times 10^{13}$ & $1.71 \times 10^{13}$ \\
\hline
\end{tabular}

It is noted that the thresholds calculated without taking into account the recombination largely underestimate the experimental thresholds, while those calculated taking into account the three-body recombination are in good agreement with the experimental values.

\subsubsection{The Results Obtained with an Excitonic Recombination Term}

In Table 2, the breakdown thresholds determined by our code are compared with the experimental measurements: the first threshold is calculated without taking into account the losses by recombination, the second one is calculated taking into account only the excitonic recombination losses.

Table 2. Comparison of the results of the breakdown code with different experimental measurements in silica in two cases: Without recombination 's and With Excitonic Recombination. All the thresholds in intensity are given in $\mathrm{W} / \mathrm{cm}^{2}$.

\begin{tabular}{|c|c|c|c|c|c|}
\hline Réf & $\lambda(\mathbf{n m})$ & $\tau$ & $\mathbf{I}_{\text {exp }}$ & I code(Without Recombination's) & I $_{\text {code }}$ (with Rec. Exciton) \\
\hline [16] & 800 & $100 \mathrm{fs}$ & $3.83 \times 10^{13}$ & $2.1 \times 10^{13}$ & $2.3 \times 10^{13}$ \\
\hline$[16]$ & 800 & $110 \mathrm{fs}$ & $3.2 \times 10^{13}$ & $1.8 \times 10^{13}$ & $2.26 \times 10^{13}$ \\
\hline$[14]$ & 800 & $140 \mathrm{fs}$ & $1.945 \times 10^{13}$ & $1.69 \times 10^{13}$ & $1.81 \times 10^{13}$ \\
\hline
\end{tabular}

In all cases, we note that the thresholds calculated taking into account the excitonic recombination are in better agreement with the experimental results compared to the results obtained with the three-body recombination.

\subsubsection{The Results Obtained by Taking into Account all the Mechanisms}

In Table 3, the thresholds of breakdown determined by our code are compared with the experimental measurements: the first threshold is calculated without taking into account the losses by recombination, the second one is calculated taking into account all the mechanisms of recombination. 
Table 3. Comparison of the results of the breakdown code with different experimental measurements in silica in two cases: Without recombination's and with all the recombination mechanisms. All intensity thresholds are given in $\mathrm{W} / \mathrm{cm}^{2}$.

\begin{tabular}{llllll}
\hline Réf & $\boldsymbol{\lambda}(\mathbf{n m})$ & $\boldsymbol{\tau}$ & $\mathbf{I}_{\text {exp }}$ & $\mathbf{I}_{\text {code }}$ (without Recombinations) & $\mathbf{I}_{\text {code }}$ (all mechanisms) \\
\hline$[16]$ & 800 & $100 \mathrm{fs}$ & $3.83 \times 10^{13}$ & $2.1 \times 10^{13}$ & $2.5 \times 10^{13}$ \\
{$[16]$} & 800 & $110 \mathrm{fs}$ & $3.2 \times 10^{13}$ & $1.8 \times 10^{13}$ & $2.28 \times 10^{13}$ \\
{$[14]$} & 800 & $140 \mathrm{fs}$ & $1.945 \times 10^{13}$ & $1.69 \times 10^{13}$ & $1.84 \times 10^{13}$ \\
\hline
\end{tabular}

In the literature much use is made of the fluence breakdown threshold - or the threshold fluence that the threshold fluence and defined as the laser fluence required for the maximum electron density obtained during the duration of the laser pulse to reach the critical electronic density of breakdown, with:

$$
F_{\text {threshold }}=\int_{-\infty}^{+\infty} I(t) d t=\frac{\tau_{p} I_{\text {threshold }}}{2}\left(\frac{\pi}{\ln 2}\right)^{1 / 2}
$$

In (Figure 8). To represent the curve of the evolution of the threshold fluence depending on the duration of the pulse obtained by our model in the case where the two recombination mechanisms are active at the same time, we note that our results are in exile agreement with the experimental results. On the other hand, it can be seen from (Figure 8) that our results confirm the Predictions of Stuart et al. on the reduction of the threshold fluence for pulses $\leq 10$ ps.

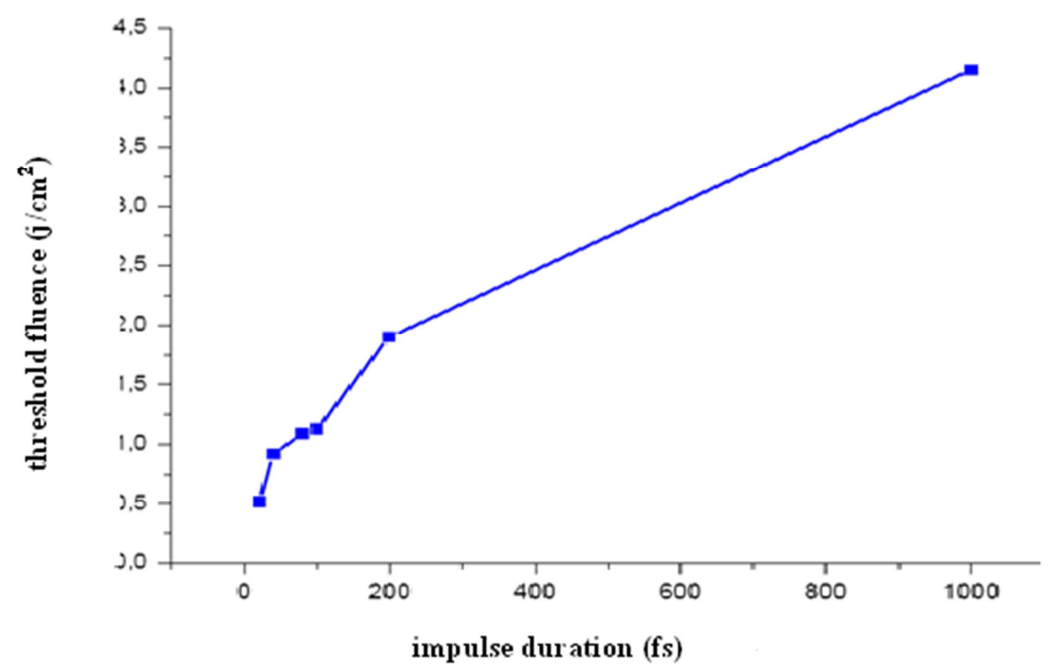

Figure 8. The evolution of the threshold fluence as a function of the duration of the pulse taking into account all the mechanisms (ionization and recombination).

\section{Influence of the Initial Electronic Density}

In this section, the influence of the initial electronic density due to the impurities on the evolution of the electron density in the conduction band and also on the threshold fluence will be studied. The results obtained are presented in (Figure 9) and (Figure 10)

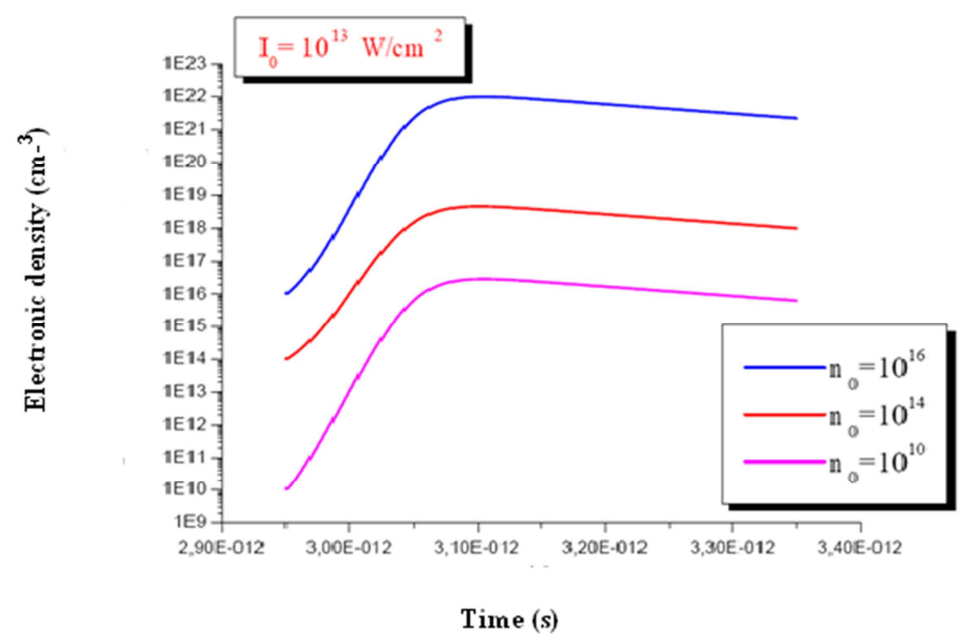

Figure 9. The influence of the initial electronic density on the evolution of the electronic density as function of the time in two cases; for: $n_{0}=10^{10} \mathrm{~cm}^{-3}$ et $n_{0}=10^{14} \mathrm{~cm}^{-3}$ et $n_{0}=10^{16} \mathrm{~cm}^{-3}$. 


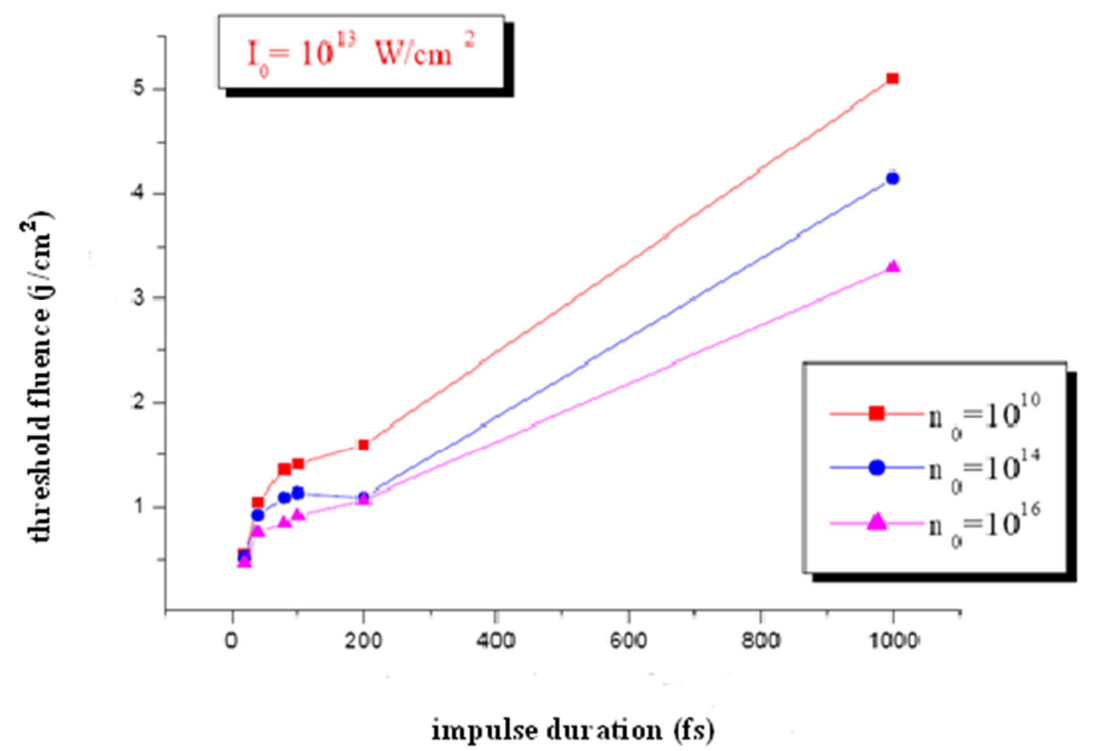

Figure 10. The influence of the initial electronic density as a function of the duration of the pulse on the threshold fluence: in the cases: $n_{0}=10^{10} \mathrm{~cm}^{-3}$ et $n_{0}=10^{14} \mathrm{~cm}^{-3}$ et $n_{0}=10^{16} \mathrm{~cm}^{-3}$.

A parameter of great practical importance is the initial electronic density from impurities in the material or generated by a pedestal of the pulses, this initial electron density may be good electron donors, which could feed and promote the AI. From (Figure 9) it is well noted the influence of the initial electronic density on the electronic density in the conduction band, when the initial electronic density is high, the electronic density in the conduction band becomes important, and consequently the decrease of the threshold fluence (Figure 10). Finally we conclude that the second term that introduced in our model also has a significant effect on the evolution of the electronic density. It is noted that in the case where the two recombination mechanisms are active, the electronic density and decrease sharply. Note that excitonic recombination is more important than 3-body recombination but it remains significant.

\section{Discussion}

A theoretical model has been developed to simulate the interaction of an ultra-short laser pulse with a dielectric. The resulting equations of the model are nonlinear and coupled differential equations, which have been solved by standard numerical methods. The scenario of this interaction can be summarized as follows:

The absorption of a photon by an electron of the levels of the heart or the valence band makes it possible to excite this electron in the conduction band. This is obviously why the incident photon has sufficient energy. Another important parameter is the laser intensity. In the case of femtosecond laser pulses, at low intensities, I $<10^{12} \mathrm{~W} / \mathrm{cm}^{2}$. The interaction is linear; the most likely process is the absorption of a single photon. For relatively larger laser intensity values this interaction becomes nonlinear and multi-photon processes have a significant probability of occurring. It is thus possible to excite an electron in the conduction band with photons whose energy is less than the width of the forbidden band.

The electrons thus excited tend to return to a situation of equilibrium: this is the recombination phase. At short time scales, it is the impact ionization that predominates for the most energetic electrons. This process corresponds to the collision of an electron of the conduction band with an electron of the valence band, with a transfer of energy allowing the latter to cross the forbidden band. For times ranging from femtosecond to picosecond the phononic processes appear, they correspond to the transfer of energy from the electron population to the crystal lattice.

The electrons arriving at the bottom of the conduction band will then recombine with a hole of the valence band by a three-body recombination mechanism or be trapped in the form of excitons.

We showed in our model that:

The recombination mechanisms reduce the electron density in the conduction band and therefore increase the optical damage threshold (OBT).

It is possible, from simple assumptions, to describe optical breakdowns in dielectric media based on the resolution of the rate equation that describes the evolution of electron density in the plasma.

The breakdown phenomena are dominated by the electronic avalanche at $100 f_{s}$, and at short durations of the laser pulse, typically $<30 f s$, it is the multi-photon ionization which becomes the dominant mechanism.

The damage threshold has a dependency on $\tau_{\mathrm{p}}^{1 / 2}$ for pulse times $\tau_{\mathrm{p}}^{1 / 2}$ ranging from $10 \mathrm{~ns}$ to $10 \mathrm{ps}$. On the other hand, when the duration of the pulses is shorter than a few picoseconds, the threshold of damage presents a change of slope tending towards a saturation for impulses $<10 \mathrm{ps}$.

The predictions of the code developed in this study have been successfully compared to different experimental measurements of thresholds of breakdown in silica [17]. 


\section{Conclusion}

The electron density in the conduction band and the optical breakdown-OBT threshold were calculated, and a comparison with the experimental measurements is made. We have found that our results are in good agreement with the experimental measurements. It has been found that the terms to be added to the equation of Stuart et al have an effect remarkable on the evolution of the electronic density and also on the OBT. Our model confirms the predictions of Stuart et al. on the reduction of the threshold fluence for pulses $\leq 10 \mathrm{ps}$. But on the other hand our model confirms the predictions of $\mathrm{Du}$ et al. On the contribution of the electronic avalanche, and that multi-photon ionization is only a source of the primary electrons of the avalanche. Finally, to treat the influence of the initial electronic density on the evolution of the electronic density in the conduction band and also on the OBT.

\section{References}

[1] G. Mourou, Appl Phys B, 65:205-211 (1997).

[2] L. V. Keldysh, Sov Phy JETP 20:1307 (1965).

[3] B. C. Stuart, M. D. Feit, S. Herman, A. M. Rubenchik, B. W. Shore, M. D. Perry, Phys Rev B, 53:1749. (1996).

[4] B. C. Stuart, M. D. Feit, S. Herman, A. M. Rubenchik, B. W. Shore, M. D. Perry J Opt Soc Am B 13: 459 (1996).

[5] G. G. Eugene and L. D. Barry, Phys Rev B 73: 214101 (2006).
[6] P. Yuri, Raizer, Gas Discharge Physics: Springer-Verlag Berlin Heidelberg (1991).

[7] Y. B. Zel'dovich and Y. P. Raizer Physics of Shock Waves and High-Temperature Hydrodynamic Phenomena, academic press New York and London (1967).

[8] L. Bergé, S. Skupin, R. Nuter, J. Kasparian, J. P. Wolf, Phys Rev Lett 92: 225002 (2004).

[9] S. S. Mao, F. Quéré, S. Guizard, X. Mao, R. E. Russo, G. petite, P. Martin, Appl Phys A 79: 1695-1709 (2004).

[10] S. Guizard, P. Martin, G. Petite, P. D’Oliveiraz, P. Meynadier, J Phys Condens Matter 8:1281-1290 (1996).

[11] P. Martin, S. Guizard, Ph. Daguzan, H. Petite, Phys Rev B 55: 5799 (1997).

[12] F. Quéré, S. Guizard, P. Martin, G. Petite, O. Gobert, P. Meynadier, M. Perdrix, Appl Phys B 68: 459-463 (1999).

[13] W. Joosen, S. Guizard, P. Martin, Appl Phys Lett 61: 2260 (1992).

[14] B. C. Stuart, M. D. Feit, S. Herman, A. M. Rubenchik, B. W. Shore, M. D. Perry, Phy Rev Lett 74: 2248 (1995).

[15] C. W. Gear Numerical Initial Value Problems in Ordinary Differential Equations, Englewood Cliffs, NJ: Prentice-Hall (1971).

[16] A. C. Tien, S. Backus, H. Kapteyn, M. Murnane, G. Mourou, Phys Rev Lett 82: 3883 (1999).

[17] O. Boultif, S. Belghit, A. Sid, interaction lasers avec un diélectriques (Lasers ultracourtes) Editions Universitaires Européennes (2017). 